\title{
Editorial
}

\section{Special Issue on Applications of Artificial Neural Networks for Energy Systems}

\author{
Giuseppe Marco Tina $\mathbb{}$ \\ Department of Electrical, Electronics and Information Engineering, University of Catania, Viale A. Doria 6, \\ 95125 Catania, Italy; giuseppe.tina@dieei.unict.it
}

Received: 3 September 2019; Accepted: 4 September 2019; Published: 7 September 2019

\section{Introduction}

In the 21st century, newer and cleaner technologies like renewable energy technologies, fuel cells, and smart grids are promising to transform the way we generate and distribute electric energy.

Long-term and ambitious commitments and stable regulatory policies have established renewable energy as a major option in the energy sector in almost all parts of the world.

For example, in 2018, within a climate and energy framework that applies until 2030, the EU decided to develop a clean, cheap, and reliable energy system. To this end, greenhouse gas emissions must decrease by at least 40\% compared to 1990 levels, and renewable energy sources like wind and solar must provide $32 \%$ of our energy. The global goal for renewable energy translates into a $57 \%$ share of renewable energy in the European energy sector by 2030, which implies that wind and solar will reshape the European energy system. Such a target for greenhouse gases will impact drastically the presence of thermoelectric power plants, as coal demand will be cut by two thirds, and oil and gas demand will be cut by a quarter.

However, seamless integration of renewables with the grids poses significant challenges due to their intermittent generation, which is intensified by the uncertainties and fluctuations existing on the demand side.

A solution is increasing energy storage and standby power generation. Alternatively, improving the predictability of wind and solar energy and demand can replace such expensive hardware with advanced control and optimization systems. In line with the increasing complexity of modern energy systems, where data are massive and inherently noisy, applications of neural-networks in solving energy problems will assume a crucial role. On the other hand, artificial neural-networks have useful specific features, such as the ability to learn the key information patterns within a multi-dimensional information domain, being fault-tolerant, robust, and possessing noise-immunity [1].

\section{Artificial Neural Networks for Energy Systems}

In light of the above, this special issue was introduced to collect latest research on relevant topics, and more importantly, to address present challenging issues with the applications of Artificial Neural Networks (ANNs) for energy systems, with specific attention to the power systems. There were 38 papers submitted to this special issue, and 12 papers were accepted (i.e., a 29\% acceptance rate). Various topics have been addressed in this special issue, mainly on non-programmable renewables (e.g., photovoltaic, wind and wave technologies) but also on batteries, fuel cells, biofuel and, the Internet of energy.

The first two papers regard the application of ANNs to transmission systems [2] and weak connected or stand-alone distribution systems [3].

Specifically paper [2] is about the reduction of occurrences of emergency situations in large-scale interconnected power systems with large continuous disturbances which are mainly caused by 
renewables. A preventive strategy for the automatic generation control (AGC) of power systems is proposed, but to mitigate the curse of dimensionality that arises in conventional reinforcement learning algorithms, a deep forest reinforcement learning (DFRL) method is proposed as a preventive strategy for AGC.

Paper [3] is about the design of a power management system for a stand-alone rural microgrid that is able to forecast both the local load demand and the generation from renewable sources in order to control the fossil fuel-based generators [3]. To address this problem, a hybrid machine learning algorithm is proposed. It divides the task of power load prediction into two sequential sub-models: the first model predicts the future weather conditions and the second model is trained to associate the predicted weather conditions with the load demand.

In this special issue, to confirm the crucial role of PVs in development of future power systems, there are three papers focused on photovoltaic (PV) systems, and forecasting as the main problem faced for best use of these systems.

The first paper [4], is about the use of ANNs to estimate and forecast solar radiation. Global horizontal irradiation (GHI) data are rarely measured worldwide, thus an artificial neural network (ANN) model is built to calculate this data based on available data. For the estimation of 5-min GHI, the normalized root mean square error (nRMSE) of the 6-inputs model is $19.35 \%$. A second ANN model was developed to transform GHI into global tilted irradiation (GTI). The GTI calculation from GHI was realized with an nRMSE of around $8 \%$ for the optimal configuration.

$\mathrm{PV}$ power generation widely varies due to environmental factors, meaning the accurate forecasting of PV generation is essential. To achieve this, ANN models were developed to forecast hourly direct normal (DNI) and GHI irradiations for time horizons varying from one hour $(h+1)$ to six hours $(h+6)$. The forecasting of hourly solar irradiation from $\mathrm{h}+1$ to $\mathrm{h}+6$ using ANN was realized with an nRMSE from $22.57 \%$ for $\mathrm{h}+1$ to $34.85 \%$ for $\mathrm{h}+6$ for GHI, and from $38.23 \%$ for $\mathrm{h}+1$ to $61.88 \%$ for $\mathrm{h}+6$ for DNI.

In the second paper [5], an adaptive solar power forecasting (ASPF) method for precise solar power forecasting, which captures the characteristics of forecasting errors and revises the predictions accordingly by combining data clustering, variable selection, and neural networks is proposed. The applied solution used improved k-means clustering, the least angular regression algorithm, and BPNN, followed by the realization of ASPF, which is shown to improve as more data is collected and used.

Missing input values reduce the PV power generation prediction performance. In reference [6], the impact of missing data imputation methods that can be used to replace these missing values is explored. We applied four missing data imputation methods to the training data and test data of the prediction model based on support vector regression. When the k-nearest neighbour's method is applied to the test data, the prediction performance yields results closest to those for the original data with no missing values, and the prediction model's performance is stable even when the missing data rate increases. Therefore, we conclude that the most appropriate missing data imputation for application to PV forecasting is the KNN method.

The inherently intermittent and highly variable nature of wind necessitates the use of wind power forecasting tools in order to facilitate the integration of wind turbines into electrical grids, two papers about this problem are present in this special issue. Specifically, in reference [7] a wind speed statistical forecasting method based on the EEMD-FuzzyEn-LSTMNN model is proposed to improve forecasting accuracy. It is divided in three steps: (1) the original wind speed series is decomposed into a series of components by using EEMD; (2) the FuzzyEn is used to calculate the complexity of each component, and the components with similar FuzzyEn values are classified into one group; (3) the LSTMNN model is used to forecast each subsequence after classification.

The forecasting result of the original wind speed series is obtained by aggregating the forecasting result of each subsequent event.

The development of a short-term wind power forecasting model based on ANN clustering, which uses statistical feature parameters in the input vector, while an enhanced version of this approach 
that adjusts the ANN output with the probability of lower misclassification (PLM) method is proposed in [8]. Moreover, it employs the Monte Carlo simulation to represent the stochastic variation of wind power production and assess the impact of energy management decisions in a residential wind-battery microgrid using the proposed wind power forecasting models.

Energy harvested from a Wave Energy Converter (WEC) varies greatly with the location of its installation. Determining an optimal location that can result in maximum output power is therefore critical. In reference [9], a novel approach to predicting the output power of a near shore WEC by characterizing ocean waves using floating buoys is presented. Experimental data are utilized to train and test prediction models. The models were developed using machine learning algorithms: SVM, RF, and ANN. The results of the experiments shows that measurements from the data collection module can yield a reliable predictor of output power. Furthermore, it found that the predictors work better when the regressors are combined with a classifier. The proposed prediction model could be used in both locating optimal placement for WECs and designing the shape of the buoys used by them.

Solid Oxide Fuel Cells (SOFCs) are promising candidates to coach the energy transition thanks to their fuel flexibility. However, SOFC performance are markedly affected by fuel composition and operative parameters. In order to optimize SOFC operation and to provide a prompt regulation, reliable performance simulation tools are required. Given the high variability ascribed to the fuel in the wide range of SOFC applications and the high non-linearity of electrochemical systems, the implementation of artificial intelligence techniques, like Artificial Neural Networks (ANNs), is sound. In reference [10], several network architectures based on a feedforward-backpropagation algorithm are proposed and trained on experimental data-set issued from tests on commercial NiYSZ/8YSZ/LSCF anode supported planar button cells.

Remaining useful life (RUL) is the useful life left on an asset at a particular time of operation. Its estimation is central to condition based maintenance and prognostics and health management. RUL is typically random and unknown, and as such it must be estimated from available sources of information such as the information obtained from conditions and health monitoring. On-line RUL prognosis is a crucial problem for aerospace Lithium-ion (Li-ion) batteries. In reference [11], while aiming to achieve practical and precise prediction of the RUL of satellite Li-ion batteries, a dynamic long short-term memory (DLSTM) neural-network-based indirect RUL prognosis was proposed. Firstly, an indirect HI based on the Spearman correlation analysis method is extracted from the battery discharge voltages, and the relationship between the indirect $\mathrm{HI}$ indices and battery capacity is established using a polynomial fitting method. Then, by integrating the Adam method, L2 regularization method, and incremental learning, a DLSTM method is proposed and applied for Li-ion battery RUL prognosis.

One paper in this special issue is about Biobutanol, which may be used as a fuel in an internal combustion engine. The most common method of producing biobutanol is the fermentation of simple sugars in biomass feedstock. In reference [12], a methodology for optimizing the biobutanol production process via the integrated acetone-butanol-ethanol (ABE) fermentation-membrane pervaporation process is proposed. In this study, artificial neural networks (ANNs) are used as metamodels in an attempt to reduce the time needed to circumscribe the Pareto domain and identify the best optimal operating conditions.

Finally, the concept of the Internet of energy (EI) has been proposed as a new high-efficiency energy structure that allows the full benefits of multi-energy coupling to be exploited.

In order to adapt to the multi-energy coupled energy structure and achieve flexible conversion and interaction of multi-energy, the concept of energy routing centers (ERCs) is proposed. In reference [13], a two-layered structure of an ERC is established. Multi-energy conversion devices and connection ports with monitoring functions are integrated in the physical layer which allows multi-energy flow with high flexibility. In order to improve the efficiency and reduce operating cost and environmental cost of the proposed EI, an artificial neural network (ANN)-based reinforcement learning algorithm is proposed to manage the optimal energy routing path. 


\section{Future Energy System and Artificial Intelligence}

To allow the management of the growing complexity of future renewable-based power systems, it is assumed that new algorithms for forecasting, managing, and controlling of both energy resources and demand, will have to be proposed and tested. The new large-scale and complex models that are required to study the future power and energy system under revolutionary paradigms (e.g., smart and micro grids, Vehicle-To-Grid V2G, and so on) cannot be solved in due time without the support of new forms of intelligent simulation and advanced optimization approaches.

Acknowledgments: This issue would not be possible without the contributions of various talented authors, hardworking and professional reviewers, and dedicated editorial team of Applied Sciences. Congratulations to all authors-no matter what the final decisions of the submitted manuscripts were, the feedback, comments, and suggestions from the reviewers and editors helped the authors to improve their papers. We would like to take this opportunity to record our sincere gratefulness to all reviewers. Finally, we place on record our gratitude to the editorial team of Applied Sciences with special mention to Daria Shi (MDPI Branch Office, Beijing) for her great attention and enthusiasm in following this project.

Conflicts of Interest: The author declares no conflict of interest.

\section{References}

1. Kalogirou, A.S. Applications of artificial neural-networks for energy systems. Appl. Energy 2006, 67, 17-35. [CrossRef]

2. Yin, L.; Zhao, L.; Yu, T.; Zhang, X. Deep Forest Reinforcement Learning for Preventive Strategy Considering Automatic Generation Control in Large-Scale Interconnected Power Systems. Appl. Sci. 2018, 8, 2185. [CrossRef]

3. Cenek, M.; Haro, R.; Sayers, B.; Peng, J. Climate Change and Power Security: Power Load Prediction for Rural Electrical Microgrids Using Long Short Term Memory and Artificial Neural Networks. Appl. Sci. 2018, 8, 749. [CrossRef]

4. Notton, G.; Voyant, C.; Fouilloy, A.; Duchaud, J.; Nivet, M. Some Applications of ANN to Solar Radiation Estimation and Forecasting for Energy Applications. Appl. Sci. 2019, 9, 209. [CrossRef]

5. Wang, Y.; Zou, H.; Chen, X.; Zhang, F.; Chen, J. Adaptive Solar Power Forecasting based on Machine Learning Methods. Appl. Sci. 2018, 8, 2224. [CrossRef]

6. Kim, T.; Ko, W.; Kim, J. Analysis and Impact Evaluation of Missing Data Imputation in Day-ahead PV Generation Forecasting. Appl. Sci. 2019, 9, 204. [CrossRef]

7. Qin, Q.; Lai, X.; Zou, J. Direct Multistep Wind Speed Forecasting Using LSTM Neural Network Combining EEMD and Fuzzy Entropy. Appl. Sci. 2019, 9, 126. [CrossRef]

8. Genikomsakis, K.; Lopez, S.; Dallas, P.; Ioakimidis, C. Simulation of Wind-Battery Microgrid Based on Short-Term Wind Power Forecasting. Appl. Sci. 2017, 7, 1142. [CrossRef]

9. Deberneh, H.; Kim, I. Predicting Output Power for Nearshore Wave Energy Harvesting. Appl. Sci. 2018, 8, 566. [CrossRef]

10. Baldinelli, A.; Barelli, L.; Bidini, G.; Bonucci, F.; Iskenderoğlu, F. Regarding Solid Oxide Fuel Cells Simulation through Artificial Intelligence: A Neural Networks Application. Appl. Sci. 2019, 9, 51. [CrossRef]

11. Wang, C.; Lu, N.; Wang, S.; Cheng, Y.; Jiang, B. Dynamic Long Short-Term Memory Neural-NetworkBased Indirect Remaining-Useful-Life Prognosis for Satellite Lithium-Ion Battery. Appl. Sci. 2018, 8, 2078. [CrossRef]

12. Elmeligy, A.; Mehrani, P.; Thibault, J. Artificial Neural Networks as Metamodels for the Multiobjective Optimization of Biobutanol Production. Appl. Sci. 2018, 8, 961. [CrossRef]

13. Wang, D.; Sun, Q.; Li, Y.; Liu, X. Optimal Energy Routing Design in Energy Internet with Multiple Energy Routing Centers Using Artificial Neural Network-Based Reinforcement Learning Method. Appl. Sci. 2019, 9, 520. [CrossRef]

(C) 2019 by the author. Licensee MDPI, Basel, Switzerland. This article is an open access article distributed under the terms and conditions of the Creative Commons Attribution (CC BY) license (http://creativecommons.org/licenses/by/4.0/). 